\title{
Acupuncture Treatment to Reduce the Risk of Dementia in Patients With Migraine: A Propensity Score-Matched Cohort Study
}

\section{Cheng-Hao Huang}

China Medical University

Mei-Chen Lin

China Medical University Hospital

I-Ching Chou

China Medical University

Ching-Liang Hsieh ( $\nabla$ clhsieh@mail.cmuh.org.tw)

China Medical University

\section{Research article}

Keywords: Acupuncture, Migraine, Dementia, National Health Insurance Research Database (NHIRD), propensity score, cohort

Posted Date: October 11th, 2021

DOI: https://doi.org/10.21203/rs.3.rs-910563/v1

License: (1) This work is licensed under a Creative Commons Attribution 4.0 International License. Read Full License 


\section{Abstract}

Background

Migraine is a recurrent headache disease that has been identified as a risk factor for subsequent dementia. In Taiwan, some patients with migraine receive acupuncture treatment for other illnesses. Therefore, the association between the effects of acupuncture treatment and the risk of dementia in patients with migraine warrants investigation. The present study collected data from Taiwan's National Health Insurance Research Database (NHIRD) to investigate the incidence of dementia in patients with migraine who did and did not concurrently receive acupuncture treatment.

Methods

We conducted a retrospective matched-cohort study that included 37,266 patients, selected from the NHIRD, who were newly diagnosed with migraine at some time between 2000 and 2012 . The follow-up period ranged from the index date (the date when patients first received acupuncture after their migraine diagnosis) to dementia diagnosis, withdrawal from the insurance program, or December 31, 2013. A 1:1 propensity score method was used to match an equal number of patients $(N=11,280)$ in the acupuncture and nonacupuncture cohorts based on sex, age, migraine diagnosis year, index year, insurance amount, urbanization level, baseline comorbidities, and medication usage. We employed Cox proportional hazards models to evaluate the risk of dementia. The cumulative incidence of dementia in both cohorts was estimated using the Kaplan-Meier method, and the difference was assessed through a log-rank test.

Results

Patients with migraine who received acupuncture treatment were found to have a lower risk of dementia (adjusted hazard ratio $[\mathrm{aHR}]=0.48,95 \% \mathrm{Cl}=0.40-0.57$ ) than those who did not undergo acupuncture treatment, after adjusting for age, sex, insurance amount, urbanization level, baseline comorbidities, and medication usage. The cumulative incidence of dementia was significantly lower in the acupuncture cohort than in the nonacupuncture cohort (log-rank test, $p<0.001)$.

Conclusions

This propensity score-matched cohort study demonstrated an association between acupuncture treatment and dementia development in patients with migraine in Taiwan. The results suggest that acupuncture treatment significantly reduced the development of dementia in patients with migraine. However, future study is required to provide more empirical evidence.

\section{Background}

Migraine is one of the most common neurological disorders; the newest edition (third) of the International Classification of Headache Disorders (ICHD-3) defines migraine as a recurrent headache disease, manifesting as a unilateral throbbing headache with moderate to severe pain intensity [1]. Common symptoms include headache and accompanying nausea, vomiting, photophobia, and occasional sensitivity to taste and sound [1]. According to a survey, the prevalence rate of migraine in the Taipei area for people over 15 years old is approximately $9.1 \%$. Among this group, the prevalence rate is $4.5 \%$ for men and $14.4 \%$ for women [2]. In a study from the United States, 
the prevalence of migraine was $8.6 \%$ in males, $17.5 \%$ in females, and $13.2 \%$ in the overall population; migraine exhibited a bimodal distribution in both sexes (peaking in those in late adolescence or their 20s and in those approximately 50 years of age) [3].

Several studies have indicated that migraine is associated with increased risks of stroke [4], depression [5], and dementia [6]. In addition, a cross-sectional prevalence study found that patients with migraine with and without aura are at increased risk for subclinical brain lesions [7]. Previous studies have revealed that silent brain lesions in the general population double the risk of dementia [8].

According to the 2019 Alzheimer's Disease International (ADI) Global Dementia Report, more than 50 million people worldwide are estimated to have dementia, and this number is expected to grow to 152 million by 2050 [9]. Dementia is a disease in which brain function is gradually lost. It not only leads to loss of memory but also adversely affects other cognitive functions, including language ability, spatial awareness, computing power, judgment, abstract thinking, and attention span. Acupuncture, traumatology manipulative therapies, and Chinese herbal products (CHPs) are all categorized as forms of traditional Chinese medicine (TCM), which is a popular form of alternative medicine in many countries [10]. Several studies have reported that acupuncture or CHPs are often used to treat migraine $[11,12]$. A population-based cohort study indicated a decreased risk of dementia in patients with migraine who used CHPs [13]. However, this study investigated the effects of CHPs only, and the potential benefits of acupuncture as preventive treatment for dementia in patients with migraine are yet unknown.

The National Health Insurance Research Database (NHIRD) of Taiwan is used for health policy and management, disease treatment research, drug efficacy and disease risk factor analysis, real-time tracking of epidemics, and medical quality improvement. The National Health Insurance (NHI) program was launched in 1995, and by the end of 2010, more than $99 \%$ of people in Taiwan were enrolled in the program [14]. A previous study reported that middle-aged people in Taiwan are most likely to receive acupuncture treatment. Other research has revealed that patients most often receive acupuncture to treat physical injury and diseases of the musculoskeletal system and of connective tissue [15]. Another study found migraine to be associated with vascular risk factors and reduced cerebral blood flow (CBF) to be a potential cause of dementia [16]. One clinical trial indicated that CBF was enhanced through acupuncture [17]. In the present study, we hypothesized that acupuncture could reduce the risk of developing dementia among patients with migraine. Therefore, the association between acupuncture treatment and the risk of dementia was evaluated in 22,560 patients with migraine between 2000 and 2012 who were identified in the NHIRD.

\section{Methods}

\section{Data source}

The NHIRD, a large-scale health database in Taiwan, has enrolled over $99 \%$ of the population of Taiwan. The database contains data on outpatient visits, hospitalizations, medical treatments, and other medical services at each hospital visit.

We conducted the analyses by Longitudinal Health Insurance Database 2000 (LHID 2000), which is a subset of the NHIRD containing 1 million individuals randomly selected from the NHIRD; that the distribution of age and sex is similar to the original database after sampling has been confirmed. For personal information protection, patient identification numbers are encrypted before being released for use. 
Each diagnosis was coded based on the International Classification of Diseases, Ninth Revision, Clinical Modification (ICD-9-CM). The study was approved by the Research Ethics Committee of China Medical University and Hospital in Taiwan [CMUH104-REC2-115(CR-6)].

\section{Study population}

This study included patients with migraine who had at least two outpatient visits or one hospitalization for migraine (ICD-9-CM: 346). Included patients were then classified into the acupuncture cohort or nonacupuncturetreated cohort.

The index date was set as the date when patients first received acupuncture treatment, and patients were followed until they were newly diagnosed as having dementia (ICD-9-CM: 290, 294.1, 331.0; at least two outpatient visits or one hospitalization), withdrew from the NHIRD, or December 31, 2013.

Migraine patients who were diagnosed with dementia before the index date, who were younger than 20 years old, or who were missing demographic information were excluded from this study. Each acupuncture-treated patient was matched with a nonacupuncture-treated patient through propensity score matching. The matching variables included age, sex, insurance amount, urbanization level, baseline comorbidities, and medication usage (Fig. 1).

Baseline comorbidity was defined as a comorbidity present before the index date and coded in the patient's medical records for at least two outpatient visits or one hospitalization. Included diseases were diabetes mellitus (ICD-9-CM: 250), hypertension (ICD-9-CM: 401-405), hyperlipidemia (ICD-9-CM: 272), coronary artery disease (ICD9-CM: 410-414), head injury (ICD-9-CM: 850-854, 959.01), chronic kidney disease (ICD-9-CM: 403-404, 585589), stroke (ICD-9-CM: 430-438), and depression (ICD-9-CM: 296.2, 296.3, 296.82, 300.4, 309.0, 309.1, 309.28, 311). Medication usage was defined as the first prescribed medication after migraine and included sumatriptan, rizatriptan, ergotamine, naproxen, diclofenac, caffeine, acetaminophen, etoricoxib, celecoxib, and ibuprofen. Patient medication usage was classified into groups taking 0,1 , 2, or 3 + medications.

\section{Statistical analysis}

The differences between categorical and continuous variables of the two cohorts were tested using the chi-square test and $t$ test, respectively.

The association between acupuncture treatment and the risk of dementia among patients with migraine was evaluated using the Cox proportional hazards model, and hazard ratios (HR), adjusted hazard ratios (aHR), and 95\% Cls were employed to estimate the risk of dementia. The incidence rate of dementia was expressed as the number of diagnoses per 1,000 person-year. The Kaplan-Meier method was applied to create cumulative incidence curves for the acupuncture-treated and nonacupuncture-treated groups. The difference between two incidence curves was determined using a log-rank test. All statistical analyses were performed using SAS, version 9.4 (SAS Institute, Cary, NC, USA). The cumulative incidence curve was plotted using R software. A two-sided $p$ value $<0.05$ indicated statistical significance.

\section{Results}

Of the 11,280 patients with migraine who received acupuncture treatment, $73 \%$ were female and the overall mean age was 46 years (Table 1). The distributions of sex, age, insurance amount, and comorbidities exhibited 
nonsignificant differences between the groups $(p>0.05)$. Patients who received acupuncture treatment lived in more urbanized areas $(p=0.005)$ and exhibited a higher rate of medication usage for migraine $(p=0.031)$. 
Table 1

Characteristics of newly diagnosed patients with migraine who did or did not receive acupuncture

\begin{tabular}{|c|c|c|c|}
\hline \multirow[t]{3}{*}{ Variable } & \multicolumn{2}{|c|}{ Accepted acupuncture } & \multirow{3}{*}{$\begin{array}{l}p \\
\text { value }\end{array}$} \\
\hline & No $(n=11280)$ & Yes $(n=11280)$ & \\
\hline & $\begin{array}{l}\text { n (\%) / Mean } \\
\text { (SD) }\end{array}$ & $\begin{array}{l}\text { n (\%) / Mean } \\
\text { (SD) }\end{array}$ & \\
\hline Sex & & & 0.893 \\
\hline Women & $8197(72.7)$ & $8206(72.7)$ & \\
\hline Men & $3083(27.3)$ & $3074(27.3)$ & \\
\hline \multicolumn{4}{|l|}{ Age at baseline } \\
\hline $20-39$ & $4363(38.7)$ & $4143(36.7)$ & \\
\hline $40-65$ & $5323(47.2)$ & $5724(50.7)$ & \\
\hline$>65$ & $1594(14.1)$ & $1413(12.5)$ & \\
\hline Mean \pm SD $(\text { years })^{b}$ & $46.4(15.8)$ & $46.3(14.8)$ & 0.621 \\
\hline Insurance amount (NT\$) & & & 0.175 \\
\hline $0-15840$ & $5293(46.9)$ & $5299(47)$ & \\
\hline $15841-28800$ & $4456(39.5)$ & $4432(39.3)$ & \\
\hline $28801-45800$ & $1093(9.7)$ & $1160(10.3)$ & \\
\hline$>45800$ & $438(3.9)$ & $389(3.4)$ & \\
\hline Urbanization $^{\mathrm{c}}$ & & & 0.005 \\
\hline 1 (highest) & $3244(28.8)$ & $3308(29.3)$ & \\
\hline 2 & 3535 (31.3) & $3392(30.1)$ & \\
\hline 3 & 1769 (15.7) & 1939 (17.2) & \\
\hline 4 (lowest) & $2732(24.2)$ & $2641(23.4)$ & \\
\hline \multicolumn{4}{|l|}{ Baseline Comorbidity } \\
\hline Diabetes mellitus & $1710(15.2)$ & $1699(15.1)$ & 0.838 \\
\hline Hypertension & 3534 (31.3) & $3513(31.1)$ & 0.763 \\
\hline Hyperlipidemia & 3135 (27.8) & 3133 (27.8) & 0.976 \\
\hline Coronary artery disease & $2206(19.6)$ & $2193(19.4)$ & 0.827 \\
\hline Head injury & $1203(10.7)$ & $1203(10.7)$ & 1.000 \\
\hline Depression & $1879(16.7)$ & $1875(16.6)$ & 0.943 \\
\hline Stroke & $1426(12.6)$ & 1382 (12.3) & 0.375 \\
\hline
\end{tabular}




\begin{tabular}{|c|c|c|c|}
\hline \multirow[t]{3}{*}{ Variable } & \multicolumn{2}{|c|}{ Accepted acupuncture } & \multirow{3}{*}{$\begin{array}{l}p \\
\text { value }\end{array}$} \\
\hline & \multirow{2}{*}{$\begin{array}{l}\text { No }(n=11280) \\
n(\%) / \text { Mean } \\
(S D)\end{array}$} & \multirow{2}{*}{$\begin{array}{l}\text { Yes }(n=11280) \\
n(\%) / \text { Mean } \\
(S D)\end{array}$} & \\
\hline & & & \\
\hline Chronic kidney disease & $1274(11.3)$ & $1272(11.3)$ & 0.966 \\
\hline Medications $^{d}$ & & & 0.031 \\
\hline 0 & $7546(66.9)$ & $7431(65.9)$ & \\
\hline 1 & $2802(24.8)$ & $2948(26.1)$ & \\
\hline 2 & $742(6.6)$ & $749(6.6)$ & \\
\hline$\geq 3$ & $190(1.7)$ & $152(1.3)$ & \\
\hline Duration between migraine date and index, days & $1147.7(989.0)$ & $1140.3(1079.7)$ & 0.595 \\
\hline \multicolumn{4}{|l|}{ Types of acupuncture, $n(\%)$} \\
\hline Manual acupuncture of TCM type & - & $9780(86.7)$ & \\
\hline Electroacupuncture & - & $353(3.1)$ & \\
\hline $\begin{array}{l}\text { Combination of manual acupuncture and } \\
\text { electroacupuncture }\end{array}$ & - & $1147(10.2)$ & \\
\hline Acupuncture visits, times & - & $8.3(18.1)$ & \\
\hline \multicolumn{4}{|l|}{ a Chi-square test } \\
\hline \multicolumn{4}{|l|}{$\mathrm{b} t$ test } \\
\hline \multicolumn{4}{|c|}{$\begin{array}{l}\text { ' Urbanization was categorized into four levels according to the population density of the residential } \\
\text { areas, with level } 1 \text { being the most urbanized and level } 4 \text { being the least urbanized }\end{array}$} \\
\hline
\end{tabular}

The mean duration of the period between the date of migraine diagnosis and the index date was more than 3 years with no significant difference between the acupuncture and nonacupuncture groups $(p=0.595)$. Most acupuncture treatments were manual acupuncture treatments $(86.7 \%)$.

Table 2 lists the potential risk factors for dementia among patients with migraine. Patients who accepted acupuncture treatment $(\mathrm{aHR}=0.48,95 \% \mathrm{Cl}=0.40-0.57)$ who resided in a relatively less urbanized area $(\mathrm{aHR}=$ $0.62,95 \% \mathrm{Cl}=0.46-0.82)$ exhibited a significantly lower risk of dementia. Patients with relatively older ages, hypertension $(\mathrm{aHR}=1.27,95 \% \mathrm{Cl}=1.02-1.59)$, stroke $(\mathrm{aHR}=1.47,95 \% \mathrm{Cl}=1.24-1.76)$, and depression $(\mathrm{aHR}=$ $1.68,95 \% \mathrm{Cl}=1.40-2.02$ ) and who used one type of migraine-related medication (aHR $=1.27,95 \% \mathrm{Cl}=1.06-$ 1.51) exhibited a significantly higher risk of dementia after adjustment for demographics, baseline comorbidities, and medication usage. 
Page 8/24 
Table 2

Cox model with hazard ratios and $95 \%$ confidence intervals of dementia associated with receiving acupuncture treatment and covariates among patients with migraine

\begin{tabular}{|c|c|c|c|c|c|}
\hline \multirow[t]{2}{*}{ Variable } & \multirow{2}{*}{$\begin{array}{l}\text { No. of } \\
\text { event } \\
(n=623)\end{array}$} & \multicolumn{2}{|l|}{ Crude } & \multicolumn{2}{|l|}{ Adjusted } \\
\hline & & $\mathrm{HR}^{\mathrm{a}}(95 \% \mathrm{Cl})$ & $\begin{array}{l}\mathrm{p}- \\
\text { value }\end{array}$ & $\mathrm{HR}^{\mathrm{b}}(95 \% \mathrm{Cl})$ & $\begin{array}{l}\mathrm{p}- \\
\text { value }\end{array}$ \\
\hline \multicolumn{6}{|c|}{ Accepted acupuncture } \\
\hline No & 425 & Ref. & & Ref. & \\
\hline Yes & 198 & $0.43(0.36-0.51)$ & $\begin{array}{l}< \\
0.001\end{array}$ & $0.48(0.40-0.57)$ & $\begin{array}{l}< \\
0.001\end{array}$ \\
\hline \multicolumn{6}{|l|}{ Sex } \\
\hline Women & 409 & Ref. & & Ref. & \\
\hline Men & 214 & $1.33(1.13-1.57)$ & $\begin{array}{l}< \\
0.001\end{array}$ & $0.93(0.79-1.11)$ & 0.431 \\
\hline \multicolumn{6}{|l|}{ Age group } \\
\hline $20-39$ & 5 & Ref. & & Ref. & \\
\hline $40-65$ & 157 & $24.17(9.92-58.86)$ & $\begin{array}{l}< \\
0.001\end{array}$ & $\begin{array}{l}20.12(8.18- \\
49.50)\end{array}$ & $\begin{array}{l}< \\
0.001\end{array}$ \\
\hline$>65$ & 461 & $\begin{array}{l}288.78(119.66- \\
696.95)\end{array}$ & $\begin{array}{l}< \\
0.001\end{array}$ & $\begin{array}{l}133.92(54.17- \\
331.12)\end{array}$ & $\hat{L}_{0.001}^{<}$ \\
\hline \multicolumn{6}{|c|}{$\begin{array}{l}\text { Insurance amount } \\
\text { (NT\$) }\end{array}$} \\
\hline $0-15840$ & 234 & Ref. & & Ref. & \\
\hline $15841-28800$ & 346 & $1.62(1.37-1.91)$ & $\begin{array}{l}< \\
0.001\end{array}$ & $1.05(0.88-1.26)$ & 0.594 \\
\hline $28801-45800$ & 36 & $0.67(0.47-0.95)$ & 0.025 & $0.91(0.64-1.31)$ & 0.624 \\
\hline$>45800$ & 7 & $0.37(0.17-0.78)$ & 0.009 & $0.58(0.27-1.24)$ & 0.162 \\
\hline \multicolumn{6}{|l|}{ Urbanization } \\
\hline 1 (highest) & 154 & Ref. & & Ref. & \\
\hline 2 & 180 & $1.08(0.87-1.34)$ & 0.491 & $0.96(0.78-1.20)$ & 0.745 \\
\hline 3 & 69 & $0.75(0.57-1)$ & 0.049 & $0.62(0.46-0.82)$ & 0.001 \\
\hline 4 (lowest) & 220 & $1.61(1.31-1.97)$ & $\begin{array}{l}< \\
0.001\end{array}$ & $0.90(0.72-1.13)$ & 0.354 \\
\hline $\begin{array}{l}\text { Baseline Como } \\
\text { (ref = nonsite } \\
\text { comorbidity) }\end{array}$ & & & & & \\
\hline
\end{tabular}




\begin{tabular}{|c|c|c|c|c|c|}
\hline \multirow[t]{2}{*}{ Variable } & \multirow{2}{*}{$\begin{array}{l}\text { No. of } \\
\text { event } \\
(n=623)\end{array}$} & \multicolumn{2}{|l|}{ Crude } & \multicolumn{2}{|l|}{ Adjusted } \\
\hline & & $\mathrm{HR}^{\mathrm{a}}(95 \% \mathrm{Cl})$ & $\begin{array}{l}\mathrm{p}- \\
\text { value }\end{array}$ & $\mathrm{HR}^{\mathrm{b}}(95 \% \mathrm{Cl})$ & $\begin{array}{l}\mathrm{p}- \\
\text { value }\end{array}$ \\
\hline Diabetes mellitus & 258 & $4.20(3.58-4.93)$ & $\begin{array}{l}< \\
0.001\end{array}$ & $1.16(0.98-1.39)$ & 0.091 \\
\hline Hypertension & 493 & $8.62(7.10-10.45)$ & $\begin{array}{l}< \\
0.001\end{array}$ & $1.27(1.02-1.59)$ & 0.033 \\
\hline Hyperlipidemia & 337 & $3.31(2.83-3.88)$ & $\begin{array}{l}< \\
0.001\end{array}$ & $0.96(0.80-1.14)$ & 0.620 \\
\hline $\begin{array}{l}\text { Coronary artery } \\
\text { disease }\end{array}$ & 376 & $6.54(5.57-7.67)$ & $<$. & $1.08(0.89-1.30)$ & 0.433 \\
\hline Head injury & 99 & $1.78(1.43-2.20)$ & $\begin{array}{l}< \\
0.001\end{array}$ & $1.17(0.94-1.45)$ & 0.168 \\
\hline Depression & 170 & $2.10(1.76-2.51)$ & $\begin{array}{l}< \\
0.001\end{array}$ & $1.68(1.40-2.02)$ & $\begin{array}{l}< \\
0.001\end{array}$ \\
\hline Stroke & 297 & $6.85(5.86-8.02)$ & $\begin{array}{l}< \\
0.001\end{array}$ & $1.47(1.24-1.76)$ & $\begin{array}{l}< \\
0.001\end{array}$ \\
\hline Chronic kidney disease & 192 & $3.68(3.10-4.36)$ & $\begin{array}{l}< \\
0.001\end{array}$ & $1.20(1.01-1.44)$ & 0.041 \\
\hline \multicolumn{6}{|l|}{ Medications } \\
\hline 0 & 269 & Ref. & & Ref. & \\
\hline 1 & 239 & $1.81(1.52-2.15)$ & $<$. & $1.27(1.06-1.51)$ & 0.009 \\
\hline 2 & 89 & $2.17(1.70-2.76)$ & $\begin{array}{l}< \\
0.001\end{array}$ & $1.08(0.85-1.38)$ & 0.531 \\
\hline$\geq 3$ & 26 & $2.41(1.60-3.61)$ & $\begin{array}{l}< \\
0.001\end{array}$ & $1.10(0.73-1.66)$ & 0.634 \\
\hline \multicolumn{6}{|c|}{$\begin{array}{l}\text { Crude } \mathrm{HR}^{\mathrm{a}} \text { represents relative hazard ratio; } \\
\text { Adjusted } \mathrm{HR}^{\mathrm{b}} \text { represents adjusted hazard ratio: mutually adjusted for receiving acupuncture, age, sex, } \\
\text { insurance amount, urbanization level, all comorbidities, and medication usage in Cox proportional hazards } \\
\text { regression }\end{array}$} \\
\hline
\end{tabular}

Table 3 displays the diagnosis distribution for patients who received acupuncture treatment. Most received treatment because of injury or poisoning $(70.1 \%)$ or receiving a diagnosis of a disease of the musculoskeletal system or connective tissue (64.8\%). 
Table 3

Distribution of acupuncture cohort by disease category or diagnosis in patients with migraine

\begin{tabular}{|c|c|}
\hline \multirow[t]{3}{*}{ Disease (ICD-9-CM) } & Acupuncture users \\
\hline & $(n=11280)$ \\
\hline & $\mathrm{n}(\%)$ \\
\hline Infectious and parasitic disease (001-139) & $27(0.2)$ \\
\hline Neoplasms (140-239) & $39(0.3)$ \\
\hline Malignant(140-208) & $24(0.2)$ \\
\hline Benign (210-229) & $16(0.1)$ \\
\hline Endocrine, nutritional and metabolic disease and immunity disorder (240-279) & $64(0.6)$ \\
\hline Blood and blood-forming organs (280-289) & $19(0.2)$ \\
\hline Mental disorder (290-319) & $118(1)$ \\
\hline Nervous system (320-389) & $967(8.6)$ \\
\hline Circulatory system (390-459) & $220(2)$ \\
\hline Respiratory system (460-519) & $491(4.4)$ \\
\hline Digestive system (520-579) & $626(5.5)$ \\
\hline Genitourinary system (580-629) & $277(2.5)$ \\
\hline Complications of pregnancy, childbirth and the puerperium (630-676) & $2(0)$ \\
\hline Skin and subcutaneous tissue (680-709) & $106(0.9)$ \\
\hline Musculoskeletal system and connective tissue (710-739) & $7305(64.8)$ \\
\hline Congenital anomalies (740-759) & $42(0.4)$ \\
\hline Certain conditions originating in the perinatal period (760-779) & $1(0)$ \\
\hline Symptoms, signs and ill-defined conditions (780-799) & $1449(12.8)$ \\
\hline Injury and poisoning (800-999) & $7904(70.1)$ \\
\hline
\end{tabular}

Several stratified analyses were conducted to verify the associations (Table 4). Patients who received acupuncture treatment exhibited a decreased risk of dementia; this was true for women $(\mathrm{aHR}=0.47,95 \% \mathrm{Cl}=$ 0.38-0.58); men $(\mathrm{aHR}=0.50,95 \% \mathrm{Cl}=0.38-0.67)$; patients aged 40 to 65 years $(\mathrm{aHR}=0.43,95 \% \mathrm{Cl}=0.31-0.61)$; patients aged over 65 years $(\mathrm{aHR}=0.44,95 \% \mathrm{Cl}=0.36-0.54)$; patients with diabetes $(\mathrm{aHR}=0.52,95 \% \mathrm{Cl}=0.40-$ 0.68), hypertension ( $\mathrm{aHR}=0.51,95 \% \mathrm{Cl}=0.42-0.61)$, hyperlipidemia $(\mathrm{aHR}=0.50,95 \% \mathrm{Cl}=0.40-0.63)$, coronary artery disease $(\mathrm{aHR}=0.47,95 \% \mathrm{Cl}=0.38-0.58)$, depression $(\mathrm{aHR}=0.51,95 \% \mathrm{Cl}=0.37-0.71)$, stroke $(\mathrm{aHR}=0.56$, $95 \% \mathrm{Cl}=0.44-0.71)$, or chronic kidney disease $(\mathrm{aHR}=0.54,95 \% \mathrm{Cl}=0.40-0.73)$; and patients with or without migraine-related medication usage $(\mathrm{aHR}=0.59,95 \% \mathrm{Cl}=0.46-0.76)$. 
Table 4. Incidence rates, hazard ratios, and confidence intervals of dementia for patients with migraine who did and did not receive acupuncture in different stratifications

Variables $\quad$ Accepted acupuncture

Compared with non-

acupuncture users

\begin{tabular}{|c|c|c|c|c|c|c|c|c|}
\hline & No & & & Yes & & & Crude HR & Adjusted \\
\hline & $(n=11$ & 80) & & $(n=11$ & 80) & & & \\
\hline & Event & $\begin{array}{l}\text { Person } \\
\text { years }\end{array}$ & $\mathrm{IR}^{\dagger}$ & Event & $\begin{array}{l}\text { Person } \\
\text { years }\end{array}$ & $\mathrm{IR}^{\dagger}$ & $(95 \% \mathrm{Cl})$ & $(95 \% \mathrm{Cl})$ \\
\hline Total & 425 & 51892 & 8.19 & 198 & 56798 & 3.49 & $\begin{array}{l}0.43(0.36- \\
0.51)^{\star \star \star}\end{array}$ & $\begin{array}{l}0.48(0.40- \\
0.57)^{\star \star \star}\end{array}$ \\
\hline
\end{tabular}

Sex

\begin{tabular}{lcccccccc} 
Women & 281 & 37082 & 7.58 & 128 & 40859 & 3.13 & $\begin{array}{l}0.41(0.34- \\
0.51)^{\star \star \star}\end{array}$ & $\begin{array}{l}0.47(0.38- \\
0.58)^{\star \star *}\end{array}$ \\
\hline Men & 144 & 14810 & 9.72 & 70 & 15940 & 4.39 & $\begin{array}{l}0.45(0.34- \\
0.6)^{\star \star *}\end{array}$ & $\begin{array}{l}0.5(0.38- \\
0.67)^{\star \star *}\end{array}$
\end{tabular}

Age group

\begin{tabular}{|c|c|c|c|c|c|c|c|c|}
\hline 20-39 & 3 & 20493 & 0.15 & 2 & 21023 & 0.10 & $\begin{array}{l}0.67(0.11- \\
4.01)\end{array}$ & $\begin{array}{l}0.46(0.07- \\
2.97)\end{array}$ \\
\hline $40-65$ & 104 & 24878 & 4.18 & 53 & 29051 & 1.82 & $\begin{array}{l}0.43(0.31- \\
0.6)^{\star \star \star}\end{array}$ & $\begin{array}{l}0.43(0.31- \\
0.61)^{\star \star \star}\end{array}$ \\
\hline$>65$ & 318 & 6521 & 48.77 & 143 & 6724 & 21.27 & $\begin{array}{l}0.44(0.36- \\
0.54)^{\star \star \star}\end{array}$ & $\begin{array}{l}0.44(0.36- \\
0.54)^{\star \star \star}\end{array}$ \\
\hline
\end{tabular}

Insurance

amount (NT\$)

\begin{tabular}{|c|c|c|c|c|c|c|c|c|}
\hline $0-15840$ & 154 & 23285 & 6.61 & 80 & 25446 & 3.14 & $\begin{array}{l}0.48(0.36- \\
0.62)^{\star \star \star}\end{array}$ & $\begin{array}{l}0.46(0.35- \\
0.61)^{\star \star \star}\end{array}$ \\
\hline $15841-28800$ & 242 & 21346 & 11.34 & 104 & 23386 & 4.45 & $\begin{array}{l}0.39(0.31- \\
0.5)^{\star \star \star}\end{array}$ & $\begin{array}{l}0.5(0.4- \\
0.63) \star \star \star\end{array}$ \\
\hline $28801-45800$ & 25 & 5246 & 4.77 & 11 & 6007 & 1.83 & $\begin{array}{l}0.39(0.19- \\
0.8)^{\star \star}\end{array}$ & $\begin{array}{l}0.31(0.15- \\
0.64)^{\star \star}\end{array}$ \\
\hline$>45800$ & 4 & 2015 & 1.99 & 3 & 1959 & 1.53 & $\begin{array}{l}0.77(0.17- \\
3.43)\end{array}$ & $\begin{array}{l}0.63(0.13- \\
3.1)\end{array}$ \\
\hline
\end{tabular}

\section{Urbanization}

\begin{tabular}{|c|c|c|c|c|c|c|c|c|}
\hline 1 (highest) & 101 & 14253 & 7.09 & 53 & 16132 & 3.29 & $\begin{array}{l}0.46(0.33- \\
0.65)^{\star \star \star}\end{array}$ & $\begin{array}{l}0.4(0.29- \\
0.56)^{\star * \star}\end{array}$ \\
\hline 2 & 124 & 16165 & 7.67 & 56 & 16812 & 3.33 & $\begin{array}{l}0.44(0.32- \\
0.6)^{\star \star \star}\end{array}$ & $\begin{array}{l}0.49(0.35- \\
0.67)^{\star \star \star}\end{array}$ \\
\hline 3 & 40 & 8411 & 4.76 & 29 & 9755 & 2.97 & $\begin{array}{l}0.63(0.39- \\
1.01)\end{array}$ & $\begin{array}{l}0.69(0.42- \\
1.13)\end{array}$ \\
\hline 4 (lowest) & 160 & 13063 & 12.25 & 60 & 14100 & 4.26 & $\begin{array}{l}0.35(0.26- \\
0.47)^{\star \star \star}\end{array}$ & $\begin{array}{l}0.47(0.35- \\
0.64)^{\star \star \star}\end{array}$ \\
\hline
\end{tabular}




\begin{tabular}{|c|c|c|c|c|c|c|c|c|}
\hline $\begin{array}{l}\text { Baseline } \\
\text { Comorbidity } \\
\text { (ref=nonsite } \\
\text { comorbidity) }\end{array}$ & & & & & & & & \\
\hline $\begin{array}{l}\text { Diabetes } \\
\text { mellitus }\end{array}$ & 171 & 7354 & 23.25 & 87 & 8292 & 10.49 & $\begin{array}{l}0.45(0.35- \\
0.59)^{\star \star \star}\end{array}$ & $\begin{array}{l}0.52(0.4- \\
0.68)^{\star \star \star}\end{array}$ \\
\hline Hypertension & 332 & 15792 & 21.02 & 161 & 17417 & 9.24 & $\begin{array}{l}0.44(0.37- \\
0.53)^{\star \star \star}\end{array}$ & $\begin{array}{l}0.51(0.42- \\
0.61)^{\star \star \star}\end{array}$ \\
\hline Hyperlipidemia & 222 & 13538 & 16.40 & 115 & 14959 & 7.69 & $\begin{array}{l}0.47(0.37- \\
0.59)^{\star \star \star}\end{array}$ & $\begin{array}{l}0.5(0.4- \\
0.63) \star \star \star\end{array}$ \\
\hline $\begin{array}{l}\text { Coronary } \\
\text { artery disease }\end{array}$ & 257 & 9662 & 26.60 & 119 & 10879 & 10.94 & $\begin{array}{l}0.41(0.33- \\
0.51)^{\star \star \star}\end{array}$ & $\begin{array}{l}0.47(0.38- \\
0.58)^{\star \star \star}\end{array}$ \\
\hline Head injury & 58 & 4978 & 11.65 & 41 & 5424 & 7.56 & $\begin{array}{l}0.65(0.44- \\
0.97)^{\star}\end{array}$ & $\begin{array}{l}0.85(0.56- \\
1.28)\end{array}$ \\
\hline Depression & 116 & 7951 & 14.59 & 54 & 8476 & 6.37 & $\begin{array}{l}0.44(0.32- \\
0.61)^{\star \star \star}\end{array}$ & $\begin{array}{l}0.51(0.37- \\
0.71)^{\star \star \star}\end{array}$ \\
\hline Stroke & 194 & 6032 & 32.16 & 103 & 6700 & 15.37 & $\begin{array}{l}0.48(0.38- \\
0.61)^{\star \star \star}\end{array}$ & $\begin{array}{l}0.56(0.44- \\
0.71)^{\star \star \star}\end{array}$ \\
\hline $\begin{array}{l}\text { Chronic kidney } \\
\text { disease }\end{array}$ & 123 & 5393 & 22.81 & 69 & 6343 & 10.88 & $\begin{array}{l}0.48(0.36- \\
0.64)^{\star \star \star}\end{array}$ & $\begin{array}{l}0.54(0.4- \\
0.73)^{\star \star \star}\end{array}$ \\
\hline \multicolumn{9}{|l|}{ Medications } \\
\hline 0 & 177 & 30432 & 5.82 & 92 & 33204 & 2.77 & $\begin{array}{l}0.48(0.37- \\
0.62)^{\star \star \star}\end{array}$ & $\begin{array}{l}0.59(0.46- \\
0.76)^{\star \star \star}\end{array}$ \\
\hline 1 & 158 & 15125 & 10.45 & 81 & 17042 & 4.75 & $\begin{array}{l}0.46(0.35- \\
0.6)^{\star \star \star}\end{array}$ & $\begin{array}{l}0.46(0.35- \\
0.61)^{\star \star \star}\end{array}$ \\
\hline 2 & 67 & 4906 & 13.66 & 22 & 5264 & 4.18 & $\begin{array}{l}0.31(0.19- \\
0.5)^{\star \star \star}\end{array}$ & $\begin{array}{l}0.35(0.21- \\
0.57)^{\star \star \star}\end{array}$ \\
\hline$\geq 3$ & 23 & 1428 & 16.11 & 3 & 1288 & 2.33 & $\begin{array}{l}0.15(0.05- \\
0.5)^{\star \star}\end{array}$ & $\begin{array}{l}0.11(0.03- \\
0.39)^{\star \star \star}\end{array}$ \\
\hline
\end{tabular}

Abbreviation: IR, incidence rate per 1,000 person-years; HR, hazard ratio; Cl, confidence interval

Adjusted $\mathrm{HR}^{\mathrm{a}}$ represented adjusted hazard ratio: mutually adjusted for receiving acupuncture, age, sex, insurance amount, urbanization level, all comorbidities, and medication usage in Cox proportional hazards regression

$\star:<0.05 ; * \star:<0.01 ; * \star \star p<0.001$

Figure 2 illustrates the significantly lower incidence of dementia in patients with migraine who underwent acupuncture treatment compared with their nonacupuncture counterparts $(p<0.001)$.

\section{Discussion}

The results of the present study indicated that the incidence of dementia was $52 \%$ lower among patients with migraine who received acupuncture treatment than among those who did not receive acupuncture treatment. This suggests that acupuncture treatment may decrease the risk of dementia in patients with migraine. After multiple 
adjustments for sex, age, insurance amount, urbanization level, baseline comorbidities, and medication usage, acupuncture treatment was found to play a key role in reducing the risk of dementia. Previous studies have reported that acupuncture can exert preventive effects in populations with several diseases associated with dementia, including traumatic brain injury (TBI) [18], stroke [19], and hypertension [20]. From this, we inferred that acupuncture may also prevent dementia; for this reason, we conducted this study.

The present study indicated that the risk of dementia increases with advancing age. Patients with migraine who were aged 40-65 years had a 20.12-fold higher risk of dementia than younger individuals, whereas the risk increased to 133.92-fold for those aged over 65 years, after adjusting for age, sex, and baseline comorbidities. The risk of developing dementia after age 60 was higher among individuals with migraine in midlife than among individuals without [21]. Therefore, aging can be identified as a key factor that increases the incidence of dementia [22].

Of all the baseline comorbidities associated with migraine, hypertension, depression, and stroke were found to significantly increase the risk of dementia the most. In previous study, patients at midlife ages with high blood pressure were found to have a higher risk of cognitive decline, including Alzheimer's disease and dementia [23]. Early life depressive symptoms have been revealed to be related to a 2 -fold or greater increase in the risk of dementia [24]. In addition, studies on stroke have revealed that vascular risk factors may also be independent risk factors for developing dementia [25]. In this study, acupuncture was found to potentially reduce the incidence of dementia in patients with hypertension, depression, and stroke.

In the current study, patients with migraine who received acupuncture treatment were divided into two groups: those affected by musculoskeletal system or connective tissue disorders (64.8\%) and those affected by injury or poisoning (70.1\%). Injury and disorders of the musculoskeletal system or connective tissue were the predominant reasons for patients to seek acupuncture treatment [15]. A previous study indicated that patients with migraine exhibited significantly more musculoskeletal dysfunction than migraine-free control participants [26]. Most patients had cervical musculoskeletal impairments that accompanied their migraine episodes [27, 28]. Acupuncture is a safe form of intervention and is a feasible option for patients with chronic neck pain [29].

A population-based database study indicated that TBI is a risk factor for migraine [30]. Acupuncture functions as a neuroprotector because it promotes the recovery of the neuronal deficit resulting from TBI by activating the brain-derived neurotrophic factor (BDNF)/tropomyosin receptor kinase B (TrkB) signaling pathway at specific acupoints (Baihui, Renzhong, Hegu, and Zusanli) [31]. Combining acupuncture with a TBI treatment regimen was reported to reduce the risk of dementia by $35 \%$ [18]. Therefore, acupuncture can not only reduce TBI symptoms but also prevent subsequent dementia.

An increased risk of dementia caused by migraine can be attributed to several mechanisms such as cerebral hypoperfusion, white matter hyperintensities (WMHs), stress, and depression [32-35]. A case report demonstrated that headache was associated with bilateral hypoperfusion starting from the occipital lobes and spreading anteriorly to the temporal and parietal lobes, providing clear evidence of the spreading depression associated with spontaneous migraine [36]. Olesen et al. [37], after observing changes in CBF, proposed that the mechanism of migraine-induced cerebral ischemia is spreading cortical depression. However, this proposition related only to migraines with aura. Another study found that, because changes in the brain indicated that cerebral small vessel disease increases the risk of most dementia types and cerebrovascular dysfunction, the vascular factors of migraine may be related to the risk of dementia [21]. Yet another study determined that acupuncture at the Buihui 
(GV20) and Yintang (GV29) points increased the CBF of the prefrontal lobe and hippocampus in mouse models of Alzheimer's disease [38]. Moreover, a pilot study demonstrated a significant increase in CBF after true acupuncture in patients with stroke [17]. Together, the results of these studies indicate that acupuncture may reduce the risk of dementia in patients with migraine, possibly by increasing CBF.

WMHs in the brain are frequently discovered on MRI scans in older people [34] and are associated with migraine [39]. High-resolution evidence has indicated that WHMs give rise to cognitive impairment and play a crucial role in the development of dementia [34, 8]. Acupuncture at the Buihui (GV20) and Zusanli (ST36) points has been found to prevent cognitive function decline in rat models of vascular dementia by ameliorating white matter perfusion and maintaining its integrity [40]. In addition, acupuncture at the Yanglingquan (GB34) point was found to potentially enhance the communication between cortices with damaged white matter tracts in patients with ischemic stroke [41]. Therefore, we infer that acupuncture modulates these alterations of white matter, thereby preventing the development of dementia.

Stress, which affects the hypothalamus-pituitary-adrenal (HPA) system, may also contribute to dementia [42, 33]. Chronic stress has been found to enhance an individual's susceptibility to inflammatory activities in the brain, which affects dementia risk by increasing inflammatory cytokines such as interleukin (IL)-1 $\beta$, IL-6, tumor necrosis factor- $a$ (TNF-a), or interferon- $\gamma$ (INF- $\gamma$ ) [33]. Oxidative stress, which induces excessive production of free radical species, could lead to oxidative biomolecules and expedite aging [43]. Electroacupuncture (EA) was found to decrease the serum levels of IL-1 $\beta$, IL-6, and TNF- $\alpha$ in a rat model of migraine [44]. In addition, acupuncture was found to attenuate cognitive impairment and neuronal death by downregulating hippocampal thioredoxininteraction proteins (TXNIPs) to inhibit oxidative stress and inflammation in a rat model of vascular dementia [45]. These studies illustrate the potential of acupuncture to protect against dementia. However, further investigation is required to determine whether these mechanisms are applicable to humans.

A national cohort study indicated that severe depression also increased the risk of dementia, with some patients being diagnosed with dementia two decades after their depressive episode [32]. Several mechanisms may link depression and dementia, including 1) vascular disease, 2) increased deposition of $\beta$-amyloid, and 3) inflammatory changes [24]. Acupuncture has been identified as having potential to enhance CBF in both animals and humans $[38,17]$. Moreover, manual acupuncture or EA at specific acupoints has been found to improve cognitive impairment and suppress the generation of amyloid beta $(A \beta)$ in several rat models of $A D$ [46]. Acupuncture also significantly decreased the serum levels of IL-1 $\beta$, IL-6, and TNF- $a$ in a chronic stress model of depression in rats [47]. Acupuncture, through multiple depression-associated pathways, could therefore reduce the risk of dementia in patients with migraine by increasing $C B F$, decreasing $A \beta$ generation, and decreasing the contents of proinflammatory cytokines in the brain.

The present study leaves open one key question: the mean number of acupuncture visits was only 8.3 during the study period, which may not be enough to prevent the development of dementia (Table 1). However, compared with standard pharmacological agents, acupuncture is a safe and beneficial treatment for chronic migraine [48]. Acupuncture not only decreases migraine recurrence for an extended period but also reduces the need for rescue medication [49]. Therefore, acupuncture may be used to improve the quality of life of and increase physical activity in patients with migraine. Moderate-intensity physical exercise, such as aerobic activity, is often prescribed as a means of decreasing cognitive impairment and reducing the risk of dementia by attenuating the progression of neurodegenerative processes and age-related loss of synapses and neuropil [50]. Notably, no more 
than 15 visits for acupuncture treatment are covered through Taiwan's NHI program per month. Patients may have received additional, self-paid acupuncture treatments. However, these treatments are not included in the NHIRD and were, therefore, not included in this study.

The present study had other limitations that should be addressed. First, the NHIRD does not indicate the severity and duration of migraine. Accordingly, we performed 1:1 propensity score matching, which was useful in minimizing the difference between the two cohorts. Second, information regarding several aspects of treatment is not recorded in the NHIRD, including the acupoints used and the frequency and duration of treatment. Therefore, we were unable to clearly identify which acupoints may treat both migraine and dementia. Third, the NHIRD does not contain laboratory data or brain images; therefore, no empirical evidence could be evaluated to understand the mechanism through which acupuncture reduces the incidence of dementia in patients with migraine. Fourth, because some cases of dementia are late onset, the duration of the current study's follow-up period was insufficient to capture these. Double-blind, randomized clinical trials are required in the future.

\section{Conclusions}

The results of the present study indicate that the incidence of dementia was lower among patients with migraine who received acupuncture treatment than among patients with migraine who did not, suggesting that acupuncture may reduce the development of dementia. This is mediated through several pathways, including the modulation of CBF, WMHs, stress, and depression. Our study emphasizes the need for clinical trials that include laboratory data and brain imaging to identify potential preventive mechanisms.

\section{Abbreviations}

ICHD-3: International Classification of Headache Disorders, Third Edition

ADI: Alzheimer's Disease International

CHPs: Chinese herbal products

TCM: Traditional Chinese medicine

NHIRD: National Health Insurance Research Database

NHI: National Health Insurance

CBF: Cerebral blood flow

LHID 2000: Longitudinal Health Insurance Database 2000

ICD-9-CM: International Classification of Disease, Ninth Revision, Clinical Modification

HR: Hazard ratio

aHR: Adjusted hazard ratio

Cls: Confidence intervals

Page $16 / 24$ 
TBI: Traumatic brain injury

AD: Alzheimer's disease

BDNF: Brain-derived neurotrophic factor

TrkB: Tropomyosin receptor kinase B

WMHs: White matter hyperintensities

HPA: Hypothalamus-pituitary-adrenal

IL: Interleukin

TNF-a: Tumor necrosis factor-a

INF-y: Interferon-y

TXNIP: Thioredoxin-interaction protein

Aß: Amyloid beta

\section{Declarations}

\section{Authors' contributions}

$\mathrm{CHH}$ conceptualized this study. MCL performed the statistical analyses. $\mathrm{CHH}, \mathrm{CLH}$, and ICC contributed to the interpretation of the acupuncture data. $\mathrm{MCL}, \mathrm{CHH}$, and ICC contributed to the interpretation of the statistical data. $\mathrm{CHH}$ drafted the manuscript. $\mathrm{CHH}, \mathrm{CLH}$, and ICC finalized the manuscript. Thus, all authors in this study have contributed to the work and have approved the manuscript for submission.

\section{Funding}

This study was supported in part by China Medical University Hospital (DMR-107-008), Taiwan Ministry of Health and Welfare Clinical Trial Center (MOHW109-TDU-B-212-114004), Multi-arm Optimization of Stroke Thrombolysis (MOST) Clinical Trial Consortium for Stroke (MOST 109-2321-B-039-002), and the Tseng-Lien Lin Foundation in Taichung, Taiwan. The study also was financially supported by the Chinese Medicine Research Center of China Medical University as part of the Featured Areas Research Center Program within the framework of the Higher Education Sprout Project by the Ministry of Education in Taiwan (CMRC-CENTER-0). The funders did not participate in carrying out the study.

\section{Availability of data and material}

Data are available from the National Health Insurance Research Database (NHIRD), published by the Taiwan National Health Insurance (NHI) Bureau. The use of the NHIRD is limited to research purposes only. Due to the legal restrictions imposed by the government of Taiwan in relation to the Personal Information Protection Act, the data used for this study cannot be made publicly available. Request for data can be sent as a formal proposal to the NHIRD (http://nhird.nhri.org.tw). 


\section{Competing interests}

The authors declare that they have no competing interests.

\section{Ethics approval and consent to participate}

The NHIRD encrypts patient personal information to protect privacy and provides researchers with anonymous identification numbers associated with relevant claims information, including sex, date of birth, medical services received, and prescriptions. Therefore, patient consent is not required to access the NHIRD. The Research Ethics Committee of China Medical University and Hospital in Taiwan approved the study [CMUH104-REC2-115(CR-6)].

\section{Consent for publication}

Not applicable.

\section{Acknowledgments}

This study was conducted using data from the NHIRD, which was provided by the National Health Insurance Administration and the Ministry of Health and Welfare, and supervised by the National Health Research Institutes. We would like to thank the management of the office for health data of China Medical University Hospital for collecting and analyzing the data used in this study.

\section{References}

1. The International Classification of Headache Disorders, 3rd edition (beta version) (2013). Cephalalgia 33 (9):629-808. doi:10.1177/0333102413485658

2. Wang SJ, Fuh JL, Young YH, Lu SR, Shia BC (2000) Prevalence of migraine in Taipei, Taiwan: a populationbased survey. Cephalalgia 20 (6):566-572. doi:10.1046/j.1468-2982.2000.00085.x

3. Victor TW, Hu X, Campbell JC, Buse DC, Lipton RB (2010) Migraine prevalence by age and sex in the United States: a life-span study. Cephalalgia 30 (9):1065-1072. doi:10.1177/0333102409355601

4. Peng KP, Chen YT, Fuh JL, Tang CH, Wang SJ (2017) Migraine and incidence of ischemic stroke: A nationwide population-based study. Cephalalgia 37 (4):327-335. doi:10.1177/0333102416642602

5. Amoozegar $F$ (2017) Depression comorbidity in migraine. International review of psychiatry (Abingdon, England) 29 (5):504-515. doi:10.1080/09540261.2017.1326882

6. Chuang CS, Lin CL, Lin MC, Sung FC, Kao CH (2013) Migraine and risk of dementia: a nationwide retrospective cohort study. Neuroepidemiology 41 (3-4):139-145. doi:10.1159/000353559

7. Kruit MC, van Buchem MA, Hofman PA, Bakkers JT, Terwindt GM, Ferrari MD, Launer LJ (2004) Migraine as a risk factor for subclinical brain lesions. Jama 291 (4):427-434. doi:10.1001/jama.291.4.427

8. Vermeer SE, Prins ND, den Heijer T, Hofman A, Koudstaal PJ, Breteler MM (2003) Silent brain infarcts and the risk of dementia and cognitive decline. The New England journal of medicine 348 (13):1215-1222. doi:10.1056/NEJMoa022066 
9. Bhatt J, Comas Herrera A, Amico F, Farina N, Wong J, Orange JB, Gaber S, Knapp M, Salcher-Konrad M, Stevens M, Australia D, Grosvenor W, Ndetei D, Baker S, Heuchemer B, Oetzel J, Banerjee S, Hill J, Oliveira D, Wattana P (2019) The World Alzheimer Report 2019: Attitudes to dementia.

10. Wang HM, Lin SK, Yeh CH, Lai JN (2014) Prescription pattern of Chinese herbal products for adult-onset asthma in Taiwan: a population-based study. Annals of allergy, asthma \& immunology : official publication of the American College of Allergy, Asthma, \& Immunology 112 (5):465-470. doi:10.1016/j.anai.2014.02.012

11. Chang YY, Tsai YT, Lai JN, Yeh CH, Lin SK (2014) The traditional Chinese medicine prescription patterns for migraine patients in Taiwan: a population-based study. Journal of ethnopharmacology 151 (3):1209-1217. doi:10.1016/j.jep.2013.12.040

12. Hsieh C-L (2012) Acupuncture as treatment for nervous system diseases. BioMedicine 2 (2):51-57. doi:https://doi.org/10.1016/j.biomed.2012.04.004

13. Liu CT, Wu BY, Hung YC, Wang LY, Lee YY, Lin TK, Lin PY, Chen WF, Chiang JH, Hsu SF, Hu WL (2017) Decreased risk of dementia in migraine patients with traditional Chinese medicine use: a population-based cohort study. Oncotarget 8 (45):79680-79692. doi:10.18632/oncotarget.19094

14. Yeh YH, Chou YJ, Huang N, Pu C, Chou P (2016) The trends of utilization in traditional Chinese medicine in Taiwan from 2000 to 2010: A population-based study. Medicine 95 (27):e4115.

doi:10.1097/md.0000000000004115

15. Wu MY, Lee YC, Lin CL, Huang MC, Sun MF, Yen HR (2018) Trends in use of acupuncture among adults in Taiwan from 2002 to 2011: A nationwide population-based study. PloS one 13 (4):e0195490.

doi:10.1371/journal.pone.0195490

16. Morton RE, St John PD, Tyas SL (2019) Migraine and the risk of all-cause dementia, Alzheimer's disease, and vascular dementia: A prospective cohort study in community-dwelling older adults. International journal of geriatric psychiatry 34 (11):1667-1676. doi:10.1002/gps.5180

17. Ratmansky M, Levy A, Messinger A, Birg A, Front L, Treger I (2016) The Effects of Acupuncture on Cerebral Blood Flow in Post-Stroke Patients: A Randomized Controlled Trial. Journal of alternative and complementary medicine (New York, NY) 22 (1):33-37. doi:10.1089/acm.2015.0066

18. Juan YH, Livneh H, Huang HJ, Lu MC, Yeh CC, Tsai TY (2019) Decreased Risk of Dementia Among Patients With Traumatic Brain Injury Receiving Acupuncture Treatment: A Population-Based Retrospective Cohort Study. The Journal of head trauma rehabilitation 34 (5):E17-e23. doi:10.1097/htr.0000000000000464

19. Chu SA, Chen TY, Chen PY, Tzeng WJ, Liang CL, Lu K, Chen HJ, Wu CC, Chen JH, Tsai CC, Wang HK (2021) Acupuncture May Decrease the Incidence of Post-stroke Dementia: A Taiwan Nationwide Retrospective Cohort Study. Frontiers in neurology 12:657048. doi:10.3389/fneur.2021.657048

20. Sun J, Ashley J, Kellawan JM (2019) Can Acupuncture Treatment of Hypertension Improve Brain Health? A Mini Review. Frontiers in aging neuroscience 11:240. doi:10.3389/fnagi.2019.00240 
21. Islamoska S, Hansen $\AA$ M, Wang HX, Garde AH, Andersen PK, Garde E, Hansen JM, Waldemar G, Nabe-Nielsen $\mathrm{K}$ (2020) Mid- to late-life migraine diagnoses and risk of dementia: a national register-based follow-up study. The journal of headache and pain 21 (1):98. doi:10.1186/s10194-020-01166-7

22. Ryan L, Hay M, Huentelman MJ, Duarte A, Rundek T, Levin B, Soldan A, Pettigrew C, Mehl MR, Barnes CA (2019) Precision Aging: Applying Precision Medicine to the Field of Cognitive Aging. Frontiers in aging neuroscience 11:128. doi:10.3389/fnagi.2019.00128

23. Sierra C (2020) Hypertension and the Risk of Dementia. Frontiers in cardiovascular medicine 7:5. doi:10.3389/fcvm.2020.00005

24. Byers AL, Yaffe K (2011) Depression and risk of developing dementia. Nature reviews Neurology 7 (6):323331. doi:10.1038/nrneurol.2011.60

25. Sahathevan R, Brodtmann A, Donnan GA (2012) Dementia, stroke, and vascular risk factors; a review. International journal of stroke : official journal of the International Stroke Society 7 (1):61-73. doi:10.1111/j.17474949.2011.00731.x

26. Luedtke K, Starke W, May A (2018) Musculoskeletal dysfunction in migraine patients. Cephalalgia 38 (5):865875. doi:10.1177/0333102417716934

27. Dodick DW (2018) Migraine. Lancet (London, England) 391 (10127):1315-1330. doi:10.1016/s01406736(18)30478-1

28. Liang Z, Galea O, Thomas L, Jull G, Treleaven J (2019) Cervical musculoskeletal impairments in migraine and tension type headache: A systematic review and meta-analysis. Musculoskeletal science \& practice 42:67-83. doi:10.1016/j.msksp.2019.04.007

29. Witt CM, Jena S, Brinkhaus B, Liecker B, Wegscheider K, Willich SN (2006) Acupuncture for patients with chronic neck pain. Pain 125 (1-2):98-106. doi:10.1016/j.pain.2006.05.013

30. Wang QR, Lu YY, Su YJ, Qin H, Zhang L, Wu MK, Zhang CL, Wu CH (2019) Migraine and traumatic brain injury: a cohort study in Taiwan. BMJ open 9 (7):e027251. doi:10.1136/bmjopen-2018-027251

31. Li X, Chen C, Yang X, Wang J, Zhao ML, Sun H, Zhang S, Tu Y (2017) Acupuncture Improved Neurological Recovery after Traumatic Brain Injury by Activating BDNF/TrkB Pathway. Evidence-based complementary and alternative medicine : eCAM 2017:8460145. doi:10.1155/2017/8460145

32. Holmquist S, Nordström A, Nordström P (2020) The association of depression with subsequent dementia diagnosis: A Swedish nationwide cohort study from 1964 to 2016 . PLoS medicine 17 (1):e1003016. doi:10.1371/journal.pmed.1003016

33. Machado A, Herrera AJ, de Pablos RM, Espinosa-Oliva AM, Sarmiento M, Ayala A, Venero JL, Santiago M, Villarán RF, Delgado-Cortés MJ, Argüelles S, Cano J (2014) Chronic stress as a risk factor for Alzheimer's disease. Reviews in the neurosciences 25 (6):785-804. doi:10.1515/revneuro-2014-0035 
34. Prins ND, Scheltens P (2015) White matter hyperintensities, cognitive impairment and dementia: an update. Nature reviews Neurology 11 (3):157-165. doi:10.1038/nrneurol.2015.10

35. Wolters FJ, Zonneveld HI, Hofman A, van der Lugt A, Koudstaal PJ, Vernooij MW, Ikram MA (2017) Cerebral Perfusion and the Risk of Dementia: A Population-Based Study. Circulation 136 (8):719-728. doi:10.1161/circulationaha.117.027448

36. Woods RP, lacoboni M, Mazziotta JC (1994) Brief report: bilateral spreading cerebral hypoperfusion during spontaneous migraine headache. The New England journal of medicine 331 (25):1689-1692.

doi:10.1056/nejm199412223312505

37. Olesen J, Friberg L, Olsen TS, Andersen AR, Lassen NA, Hansen PE, Karle A (1993) Ischaemia-induced (symptomatic) migraine attacks may be more frequent than migraine-induced ischaemic insults. Brain : a journal of neurology 116 ( Pt 1):187-202. doi:10.1093/brain/116.1.187

38. Ding N, Jiang J, Xu A, Tang Y, Li Z (2019) Manual Acupuncture Regulates Behavior and Cerebral Blood Flow in the SAMP8 Mouse Model of Alzheimer's Disease. Frontiers in neuroscience 13:37. doi:10.3389/fnins.2019.00037

39. Hamedani AG, Rose KM, Peterlin BL, Mosley TH, Coker LH, Jack CR, Knopman DS, Alonso A, Gottesman RF (2013) Migraine and white matter hyperintensities: the ARIC MRI study. Neurology 81 (15):1308-1313. doi:10.1212/WNL.0b013e3182a8235b

40. Ma SM, Wang L, Su XT, Yang NN, Huang J, Lin LL, Shao JK, Yang JW, Liu CZ (2020) Acupuncture Improves White Matter Perfusion and Integrity in Rat Model of Vascular Dementia: An MRI-Based Imaging Study. Frontiers in aging neuroscience 12:582904. doi:10.3389/fnagi.2020.582904

41. Han X, Bai L, Sun C, Niu X, Ning Y, Chen Z, Li Y, Li K, Lyu D, Fu C, Cui F, Chen Z, Tan Z, Tang L, Zou Y (2019) Acupuncture Enhances Communication between Cortices with Damaged White Matters in Poststroke Motor Impairment. Evidence-based complementary and alternative medicine : eCAM 2019:4245753. doi:10.1155/2019/4245753

42. Gradus JL, Horváth-Puhó E, Lash TL, Ehrenstein V, Tamang S, Adler NE, Milstein A, Glymour MM, Henderson VW, Sørensen HT (2019) Stress Disorders and Dementia in the Danish Population. American journal of epidemiology 188 (3):493-499. doi:10.1093/aje/kwy269

43. Bennett S, Grant MM, Aldred S (2009) Oxidative stress in vascular dementia and Alzheimer's disease: a common pathology. Journal of Alzheimer's disease : JAD 17 (2):245-257. doi:10.3233/jad-2009-1041

44. Zhao L, Liu L, Xu X, Qu Z, Zhu Y, Li Z, Zhao J, Wang L, Jing X, Li B (2020) Electroacupuncture Inhibits Hyperalgesia by Alleviating Inflammatory Factors in a Rat Model of Migraine. Journal of pain research 13:75-86. doi:10.2147/jpr.S225431

45. Du SQ, Wang XR, Zhu W, Ye Y, Yang JW, Ma SM, Ji CS, Liu CZ (2018) Acupuncture inhibits TXNIP-associated oxidative stress and inflammation to attenuate cognitive impairment in vascular dementia rats. CNS neuroscience \& therapeutics 24 (1):39-46. doi:10.1111/cns.12773 
46. Park S, Lee JH, Yang EJ (2017) Effects of Acupuncture on Alzheimer's Disease in Animal-Based Research. Evidence-based complementary and alternative medicine : eCAM 2017:6512520. doi:10.1155/2017/6512520

47. Lu J, Shao RH, Hu L, Tu Y, Guo JY (2016) Potential antiinflammatory effects of acupuncture in a chronic stress model of depression in rats. Neuroscience letters 618:31-38. doi:10.1016/j.neulet.2016.02.040

48. Zhang N, Houle T, Hindiyeh N, Aurora SK (2020) Systematic Review: Acupuncture vs Standard Pharmacological Therapy for Migraine Prevention. Headache 60 (2):309-317. doi:10.1111/head.13723

49. Zhao L, Chen J, Li Y, Sun X, Chang X, Zheng H, Gong B, Huang Y, Yang M, Wu X, Li X, Liang F (2017) The Longterm Effect of Acupuncture for Migraine Prophylaxis: A Randomized Clinical Trial. JAMA internal medicine 177 (4):508-515. doi:10.1001/jamainternmed.2016.9378

50. Ahlskog JE, Geda YE, Graff-Radford NR, Petersen RC (2011) Physical exercise as a preventive or diseasemodifying treatment of dementia and brain aging. Mayo Clinic proceedings 86 (9):876-884. doi:10.4065/mcp.2011.0252

\section{Figures}




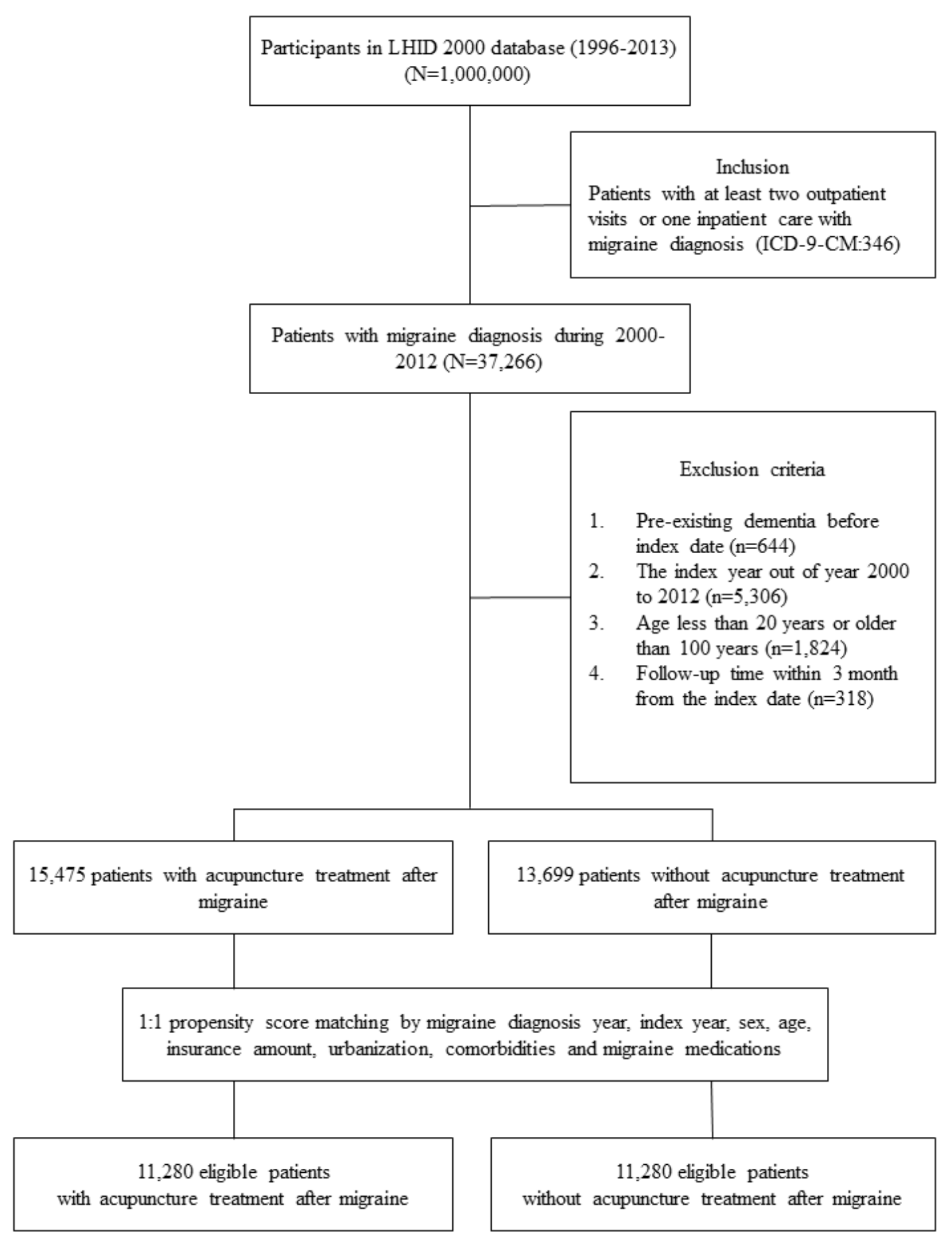

\section{Figure 1}

Study population flowchart. 37,266 patients with migraine, newly diagnosed between 2000 and 2012, were identified. A 1:1 propensity score was used to match cohorts based on sex, age, baseline comorbidities, and medication usage. The groups of acupuncture users and nonacupuncture users each contained 11,280 patients. LHID 2000: Longitudinal Health Insurance Database 2000, NHI: National Health Insurance 


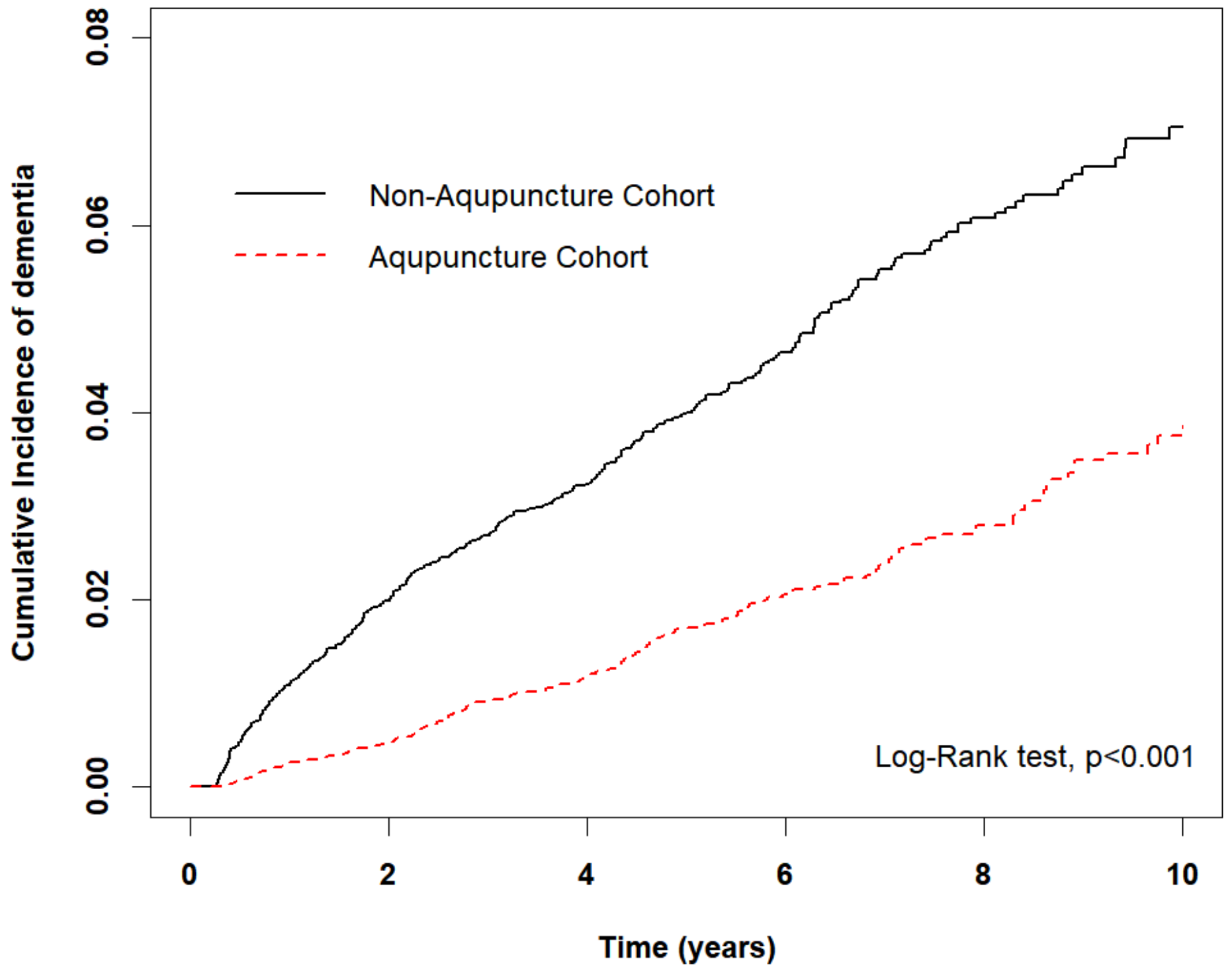

Figure 2

Cumulative incidence of dementia in the acupuncture and nonacupuncture cohorts. Cumulative incidence of dementia in the acupuncture cohort was significantly lower than in the nonacupuncture cohort (log-rank test, $p<$ 0.0001). 